\title{
Characterization of Stiffness Degradation caused by Fatigue Damage of Additive Manufactured Parts
}

\author{
C. W. Ziemian ${ }^{\text {a, }}$, R. D. Ziemian ${ }^{\mathrm{b}}$, K. V. Haile ${ }^{\mathrm{a}}$ \\ ${ }^{a}$ Bucknell University, Department of Mechanical Engineering, Lewisburg, PA 17837 \\ ${ }^{b}$ Bucknell University, Department of Civil \& Environmental Engineering, Lewisburg, PA 17837 \\ * Corresponding author. Tel.00 1 5705771754; fax 5705777281; cziemian@bucknell.edu
}

\begin{abstract}
A study of the cyclical fatigue behavior of additive manufactured components, fabricated by the fused deposition modeling (FDM) process, is presented. Experimentation was designed to focus on the effect of deposition strategy or specimen mesostructure on tensile fatigue life and effective stiffness. Testing included consideration of unidirectional laminates with parallel plies having fiber orientations ranging from $\theta=0^{\circ}$ to $\theta=90^{\circ}$, and bidirectional laminates with alternating orthogonal plies that form a layering pattern of $\theta^{\circ} /\left(\theta-90^{\circ}\right)$ fiber orientations. Results highlight the orthotropic behavior of FDM components and suggest that tensile performance is improved by aligning fibers of unidirectional laminae more closely with the axis of applied stress. The bidirectional laminae display incrementally improved tensile fatigue performance from what appears to be an offsetting effect associated with alternating orthogonal layers. An empirical model of effective elastic modulus and an analytical model of the accumulated damage state, as defined on the basis of stiffness degradation during cyclical loading, are presented as functions of specimen mesostructure. The actual damage accumulation due to cyclical loading is compared with the model predictions, and the coefficient of determination $\mathrm{R}^{2}$ indicates reasonable agreement for each factor combination.
\end{abstract}

\section{Keywords}

Additive Manufacturing; Fatigue Life; Cyclical Damage;

\section{Introduction}

Additive manufacturing (AM) processes form a class of fabrication techniques that construct components using a computerized layering process that incrementally builds one planar part slice (or layer) on top of another. These additive layering procedures, which includes the creation of any structural supports needed to accommodate overhanging features, are capable of generating components with complex geometrical shapes in a single step process [1]. Although traditionally used for rapid prototyping, AM processes have begun to evolve into rapid manufacturing methods intended to produce functional components for end use in marketable products. This advancement requires that, in addition to a durable base material, the mechanical properties of AM components sufficiently meet in-service loading and operational requirements, and are reasonably comparable to the properties of parts produced by more traditional manufacturing methods [2]. A thorough understanding of the mechanical properties and behavior of AM parts is therefore necessary; as is a reliable method for accurately predicting and controlling these properties.

Fused deposition modeling (FDM) Stratasys Inc. is an AM process that produces durable components by layering extruded materials; most commonly acrylonitrile butadiene styrene (ABS) plastic. The process involves the partial melting and extrusion of a flexible ABS filament though a heated nozzle, forming a thin, single layer pattern on the surface of the previously extruded layer. Each resulting layer (or lamina) is thus composed of closely packed, parallel, semi-solid fibers or rasters that subsequently cool, solidify, and bond with adjacent and previously deposited fibers. FDM components formed in this manner have been shown to possess anisotropic mechanical properties. The literature includes considerable attention to the directional dependence of FDM component properties, including tensile [2-7], compressive [4,7-9], flexural [5-7], and impact strengths [6,7]. A comprehensive review of this literature is presented in Ziemian et al. [10]. An important factor still limiting the use of FDM 
components within marketable products, however, is the remaining question of their fatigue response.

There is currently a lack of published work regarding the cyclical fatigue performance of FDM parts. Lee and Huang [11] performed an investigation that analyzed the total strain energy absorbed by two different FDM plastics during fatigue testing. While their work provides important baseline data, their experiment tested only one material specimen at each stress level, thereby precluding any analysis of statistical significance. Similarly, Ziemian et al. [12] completed a preliminary study of FDM fatigue properties as part of an experimental investigation of the dependence of tensile strength on the orientations of the rasters relative to the loading axis. The fatigue study included statistical samples for four mesostructures, i.e. raster orientation and layering pattern, which were each considered at four different maximum stress levels. Results and analysis of microscopic failure mechanisms indicated the superior tension fatigue performance of the bidirectional specimens with $+45 /-45^{\circ}$ layering pattern.

This paper presents results of additional experimentation and the analysis of the data, expanding upon the previous study [12] to now investigate the effect of cyclical loading on FDM specimen stiffness. The use of classical lamination theory (CLT) to predict the effective elastic modulus as a function of specimen mesostructure is first implemented on the basis of published work in the literature. The current study investigates the possibility that FDM components, similar to laminate composite materials, undergo a loss of elastic modulus and a decrease in residual strength as a result of multiple damage modes during cyclical loading [13]. The goal of this work is to gain important insight into the fatigue damage state and cyclical performance of FDM parts through the analysis of the resulting changes in the effective elastic modulus.

\section{Experimental Study}

Although a presentation of a portion of this experimental study has been previously published [12], an updated summary is provided here for the sake of completeness.

\subsection{Methods and materials}

Dogbone shaped specimens, per the ASTM D638 standard [14], were fabricated on a Stratasys Vantage-i machine of acrylonitrile butadiene styrene (ABS-P400 Stratasys $\left.{ }^{\circledR}\right)$. Specimens were built lying flat on the machine platform, with the minimum part dimension aligned with the $\mathrm{z}$-axis of the machine (Figure 1a). FDM machine process parameters were set at default or recommended values, including air gap $(0.0 \mathrm{~mm})$, road width $(0.3048 \mathrm{~mm})$, slice height $(0.1778 \mathrm{~mm})$, interior fill style (solid normal), and part fill style (perimeter/raster). A T12 nozzle was used.

Each dogbone specimen had a z-height, or thickness, of $2.54 \mathrm{~mm}$ and included a total of 15 total layers. Specimens were built with a variety of fiber orientations and layering patterns, i.e. mesostructures. Fiber orientation $\theta$ is defined as the angle between the fiber direction and the longitudinal axis of the part (Figure 1b). Specimens included seven unidirectional configurations, with 15 identical and parallel fiber orientations of $\theta=0,15,30,45,60,75$, and 90 degrees. Specimens with bidirectional laminates were also built, including four additional mesostructures with alternating orthogonal plies, i.e. $0^{\circ} /-90^{\circ},+15^{\circ} /-75^{\circ},+30^{\circ} \%-60^{\circ},+45^{\circ} /-45^{\circ}$.

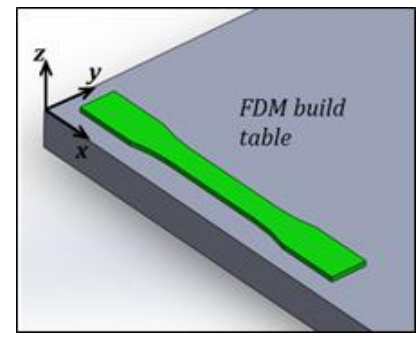

(a)

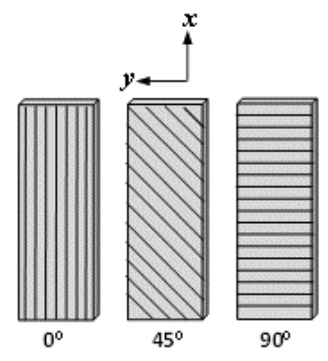

(b)

Fig. 1. Building FDM specimens; (a) Build orientation and (b) representative fiber orientations, $\theta$ [12]. 


\subsection{Monotonic tensile properties}

Tension tests were first completed, per the ASTM D638 standard [14], to determine the mean ultimate tensile stress (UTS), yield stress, and effective modulus of elasticity along the longitudinal loading direction for each mesostructure. Four specimens of each type were tested at a strain rate of $0.00065 \mathrm{~s}^{-1}$ on an Instron machine with .057 micron displacement precision, up to $0.001 \mathrm{~N}$ force accuracy, and $10 \mathrm{kN}$ load capacity. Each tension specimen was stressed until fracture occurred. Additional $0^{\circ}$ and $90^{\circ}$ specimens were affixed with strain gages to record lateral and transverse strains during tension testing, from which major and minor Poisson ratios could be estimated.

A summary of the resulting tensile strength and stiffness data is presented in Table 1. The effective elastic modulus for each specimen tested was calculated from the slope of the linear portion of the stress-strain curves, i.e. $\Delta \sigma / \Delta \varepsilon$. A MATLAB program was used to perform a least squares linear regression analysis of the data to determine the fitted slope for each specimen, from which the effective modulus was computed. The mean effective modulus reported in Table 1 data has been adjusted to account for the effective length (due to dogbone geometry). The same MATLAB program was used to compute the relationship of the slopes of two curves (stress-vs-axial strain and stress -vs-transverse strain) in estimating Poisson's ratio.

Table 1. Tension test results for various mesostructures and single fiber specimens; Standard deviation appears in parentheses;

\begin{tabular}{cccc}
\hline $\begin{array}{c}\text { Fiber } \theta \\
\text { Mesostructure }\end{array}$ & $\begin{array}{c}\text { Mean Yield } \\
\text { Strength }(\mathrm{MPa})\end{array}$ & $\begin{array}{c}\text { Mean Ultimate } \\
\text { Strength }(\mathrm{MPa})\end{array}$ & $\begin{array}{c}\text { Mean Effective } \\
\text { Modulus (MPa) }\end{array}$ \\
\hline $0^{\circ}$ & $22.82(0.36)$ & $23.08(0.30$ & $1820.54(15.41)$ \\
$15^{\circ}$ & $15.53(0.52)$ & $15.82(0.50)$ & $1722.31(20.47)$ \\
$30^{\circ}$ & $13.26(0.43)$ & $13.99(0.40)$ & $1475.52(41.40)$ \\
$45^{\circ}$ & $10.06(0.39)$ & $10.61(0.39)$ & $1231.15(28.43)$ \\
$60^{\circ}$ & $8.04(0.23)$ & $8.50(0.29)$ & $1103.40(33.77)$ \\
$75^{\circ}$ & $7.67(0.16)$ & $7.97(0.30)$ & $1105.64(41.11)$ \\
$90^{\circ}$ & $8.19(0.18)$ & $8.37(0.31)$ & $1103.24(26.90)$ \\
$+45^{\circ} / 45^{\circ}$ & $14.53(0.34)$ & $15.71(0.30)$ & $1362.28(19.97)$ \\
$+30^{\circ} / 60^{\circ}$ & $14.17(0.17)$ & $14.67(0.13)$ & $1443.83(44.58)$ \\
$+15^{\circ} /-75^{\circ}$ & $14.37(0.24)$ & $14.39(0.25)$ & $1524.28(20.44)$ \\
$0^{\circ} /-90^{\circ}$ & $14.34(0.24)$ & $14.36(0.23)$ & $1538.22(36.88)$ \\
Single fiber & $25.14(0.41)$ & $27.74(0.53)$ & $1914.48(43.23)$ \\
\hline
\end{tabular}

While the single fiber specimens were the strongest and stiffest overall, the data for the AM components indicate that the mean ultimate and yield strengths $(0.2 \%$ offset $)$ were largest for the longitudinal $\left(0^{\circ}\right)$ specimens and smallest for the transverse $\left(90^{\circ}\right)$ specimens. The mean ultimate tensile strength (UTS) of the $90^{\circ}$ specimens was only $36.4 \%$ of that of the $0^{\circ}$ raster specimens. A one-way analysis of the variance (ANOVA), and subsequent post hoc comparison, indicated a statistically significant difference between all of the UTS values for the four raster orientations at a level of significance of $\alpha=0.05$, with a calculated F-test statistic of $\mathrm{F}(3,12)=2337.46$ and a $p$-value of 0.000 . These data support that specimen tensile strength is affected by the directional processing, and raster orientation has a significant effect on the tensile strengths of the FDM specimens [10].

The monotonic tensile response observed for representative specimen types [12] indicates dissimilarities with regard to both strength and fracture strain on the basis of mesostructure.

Unidirectional specimens with $45^{\circ} \leq \theta \leq 90^{\circ}$ strained significantly less than other orientations, with only minor amounts of deformation prior to failure, and fracture occurring almost immediately after yielding. These specimens also displayed considerably smaller tensile strengths than other mesostructures, suggesting that their tensile responses are highly dependent on the inter-fiber bond strength that forms during processing. The individual fibers and the $0^{\circ} \mathrm{FDM}$ specimens, in contrast, display more significant straining prior to failure, and higher tensile strengths. They also exhibit a post yielding drop in stress that 
is symptomatic of the polymer chain stretching, rotation, and sliding in thermoplastic materials [11]. As anticipated, this suggests that the tensile response of the $0^{\circ}$ orientation is based much more significantly on the behaviour of the ABS polymer and extruded fibers themselves. Finally, the alternating $\theta^{\circ} /\left(\theta-90^{\circ}\right)$ specimens display levels of tensile strength and post-yield straining that are less than the unidirectional $\theta$ $\leq 15^{\circ}$ parts, but greater than the other unidirectional laminates.

\subsection{Cyclical tensile response}

Tension-tension fatigue tests were performed on a subset of mesostructures according to a factorial experimental design, with two factors analyzed at different levels. Factor $A$, raster orientation, included unidirectional $0^{\circ}, 45^{\circ}, 90^{\circ}$, and bidirectional $0^{\circ} /-90^{\circ},+15^{\circ} /-75^{\circ},+30^{\circ} /-60^{\circ},+45^{\circ} /-45^{\circ}$. Factor $B$, maximum stress level, included $90,75,60$, and $45 \%$ of UTS. The minimum cycle load in each case was $1 / 10$ of the maximum load (stress ratio $\mathrm{R}=0.1$ ), and tests were performed with triangular load variation in time. Tests were completed in accordance with the ASTM D7791 standard [15], with at least four replicate specimens for each factor combination. Cyclical loading was performed at room temperature in a humidity-controlled environment using a low frequency $(0.25 \mathrm{~Hz})$ to limit the possibility of localized heating due to hysteresis, and a runout value of 25 hours of testing, or approximately 17,500 cycles.

Updated fatigue life data, summarized on the basis of normalized stress (\% UTS), are presented in Table 2. Results indicate that the transverse $\left(90^{\circ}\right)$ raster specimens experience the shortest fatigue life, and the default bidirectional $\left(+45^{\circ} /-45^{\circ}\right)$ specimens had the longest life at each normalized stress level. A two-way ANOVA [16] revealed that the main effects of both stress level and raster orientation, as well as a stress-orientation interaction effect, are relevant at $\alpha=0.05$. This statistical analysis, and consideration of the variability in the data, have been previously published [12]. Results confirm that fiber orientation relative to loading axis substantially affects tension-fatigue properties, and the difference between the mean numbers of cycles to failure of each orientation is statistically significant at each stress level. The interaction between stress level and raster orientation suggests that the relative effect of fiber orientation differs for each stress level, and vice-versa. Data variability was found to increase with dependence on weaker inter-fiber bonding (i.e. $45^{\circ}$ and $90^{\circ}$ specimens), which is a result of the FDM extrusion process.

Table 2. Tension-tension fatigue results presented as the mean number of cycles to failure; Standard deviation, in units of fatigue cycles, appears in parentheses;

\begin{tabular}{|c|c|c|c|c|}
\hline \multirow{2}{*}{ Fiber $\theta$} & \multicolumn{4}{|c|}{ Maximum Loading (\% ultimate tensile strength) } \\
Mesostructure & $90 \%$ UTS & $75 \%$ UTS & $60 \%$ UTS & $45 \%$ UTS \\
\hline $0^{\circ}$ & $315(39)$ & $1738(161)$ & $4315(591)$ & $17169^{\wedge}(1158)$ \\
\hline $45^{\circ}$ & $176(29)$ & $706(149)$ & $3057(870)$ & $17843(4230)$ \\
\hline $90^{\circ}$ & $45(13)$ & $308(193)$ & $1271(228)$ & $10575(2757)$ \\
\hline$+45^{\circ} / 45^{\circ}$ & $1155(86)$ & $4592(366)$ & $13628(3463)$ & $\wedge *$ \\
\hline$+30^{\circ} / 60^{\circ}$ & $1272(163)$ & $3463(211)$ & $11547(900)$ & $\wedge^{\star *}$ \\
\hline$+15^{\circ} /-75^{\circ}$ & $809(207)$ & $2692(407)$ & $8249(1719)$ & $\wedge^{*}$ \\
\hline $0^{\circ} /-90^{\circ}$ & $468(111)$ & $3184(420)$ & $13634(841)$ & $\wedge^{*}$ \\
\hline
\end{tabular}

$\wedge$ Run at 50\% UTS; *All 50\% UTS specimens were stopped (unbroken) at runout value of 25 hours;

The logarithm of constant amplitude fatigue life was found to be linearly dependent on the logarithm of the governing stress (Figure 2). The data were transformed using the log-base 10 of both the mean number of cycles to failure $(N)$ and the maximum stress level per cycle (\%UTS). In this form, the curves have similar decreasing slopes. The positioning of the lines along the horizontal axis clearly indicates the generally superior fatigue performance of the $0^{\circ}$ and $+45^{\circ} /-45^{\circ}$ mesostructures at each \%UTS value.

Based on the transformed data, the $\mathrm{S}-\mathrm{N}$ curves can be expressed in the form of a power law:

$$
N=a S^{m}
$$

where $m$ is the fatigue exponent, $a$ is the fatigue coefficient, and $S$ is the maximum cycle stress. Although 
$N$ is the dependent variable, it is traditionally plotted on the abscissa in graphical representations of S-N curves (Figure 2). However, these axes were swapped to accurately represent the linear relationship when determining the fatigue life model parameters. Material parameters $m$ and $a$ were empirically determined from the experimental data using a least squares regression fit, and appear in Table 3.
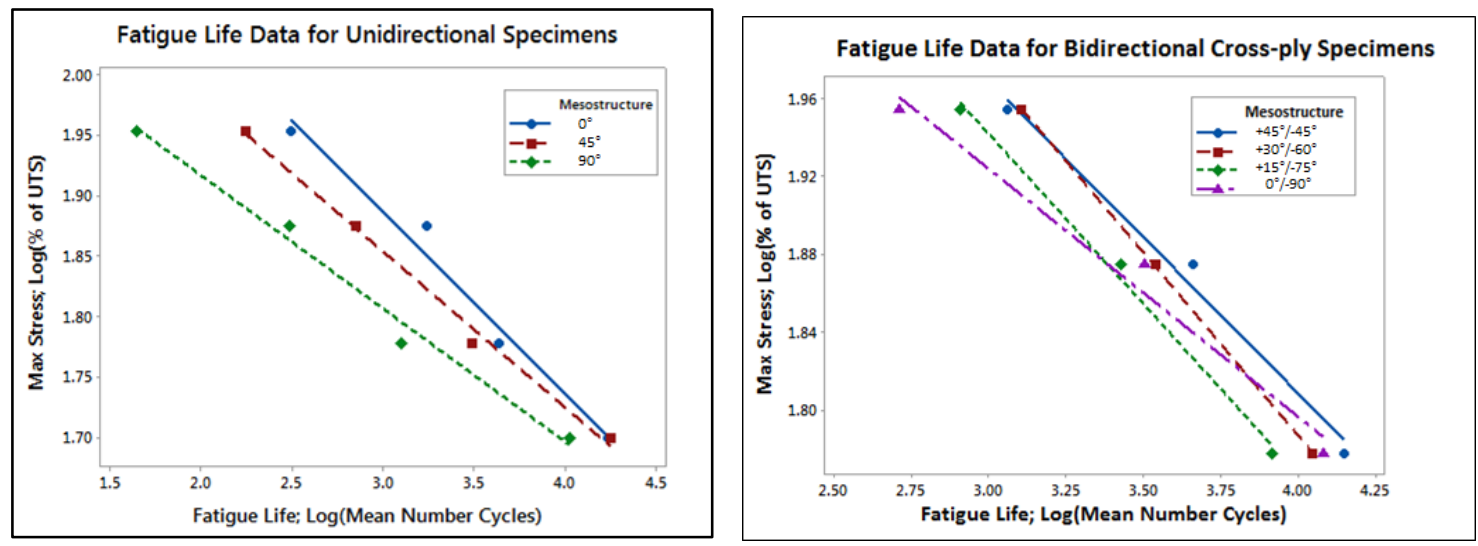

Fig. 2. Estimated S-N curves; (a) Unidirectional mesostructures at four stress levels tested; (b) Cross-ply bidirectional mesostructures at three stress levels tested;

Table 3. Estimated material parameters associated with a power law model for the fatigue life of FDM specimens $(\mathrm{R}=0.10)$;

\begin{tabular}{|c|c|c|c|}
\hline $\begin{array}{c}\text { Fiber } \theta \\
\text { Mesostructure }\end{array}$ & $\begin{array}{c}\text { Fatigue } \\
\text { Exponent } m\end{array}$ & $\begin{array}{c}\text { Fatigue } \\
\text { Coefficient } a\end{array}$ & $\begin{array}{c}\text { Regression } \\
R^{2}\end{array}$ \\
\hline $0^{\circ}$ & -6.40 & 11.24 & 0.977 \\
\hline $45^{\circ}$ & -6.62 & 8.84 & 0.998 \\
\hline $90^{\circ}$ & -7.67 & 8.78 & 0.991 \\
\hline$+45^{\circ}-45^{\circ}$ & -5.46 & 9.59 & 0.984 \\
\hline$+30^{\circ} /-60^{\circ}$ & -5.36 & 9.11 & 0.999 \\
\hline$+15^{\circ} /-75^{\circ}$ & -6.01 & 9.60 & 0.996 \\
\hline $0^{\circ} /-90^{\circ}$ & -6.79 & 10.37 & 0.976 \\
\hline
\end{tabular}

\section{Lamination Analysis of FDM Components}

Although standard FDM components are composed of a single build material (typically ABS), their mechanical properties depend on a variety of characteristics, including filament material properties, orientation of the fibers relative to loading direction, strength and coverage of the bond formed between fibers, and the volume and geometry of air gaps or voids [17]. As such, it is potentially useful to view FDM components as a form of laminate composite structure with vertically stacked layers (lamina) of bonded ABS fibers or rasters [8]. In this context, each FDM layer of parallel fibers can be assimilated to a composite material with two-phases, i.e. the thermoplastic ABS material and the air voids, for which results of previous studies indicate orthotropic material behavior [17-19]. The foundational work in this regard was completed by Kulkarni and Dutta [17], and was later expanded by Li et al. [18], who used classical lamination theory (CLT) in quantifying the relationship between deposition strategies and FDM component stiffness.

\subsection{Constitutive models}

In modeling FDM specimens as laminated composites of bonded laminars, each layer (lamina) is an orthotropic composite of ABS filaments, the bond between filaments, and the air voids. The orientation of 
parallel fibers in a single lamina is defined as the angle $\theta$ between the longitudinal axis of the filaments ( 1 -axis) and the axial loading direction ( $x$-axis). Since each lamina is a thin layer, the assumption of plane stress applies. With this and the principal coordinate axes in the plane of the lamina (1-2 plane), the stress components $\sigma_{3}, \tau_{23}$, and $\tau_{13}$ become zero and the $6 \times 6$ elastic stiffness matrix condenses to a $3 \times 3$ reduced stiffness matrix $[\mathrm{Q}]$. For an orthotropic material in which the principal material directions coincide with the loading directions (i.e. $\theta=0$ or $90^{\circ}$ ), the [Q] matrix further simplifies to the form seen in the in-plane stress-strain relationship in Eq. 3.1:

$$
\left[\begin{array}{l}
\sigma_{11} \\
\sigma_{22} \\
\sigma_{12}
\end{array}\right]=\left[\begin{array}{ccc}
Q_{11} & Q_{12} & 0 \\
Q_{12} & Q_{22} & 0 \\
0 & 0 & Q_{66}
\end{array}\right]\left[\begin{array}{l}
\epsilon_{11} \\
\epsilon_{22} \\
\epsilon_{12}
\end{array}\right]
$$

A convenient form of [Q] for this specially orthotropic case (i.e. $\theta=0$ or $90^{\circ}$ ) is provided by defining its elements in terms of Young's modulus in longitudinal $\left(\mathrm{E}_{11}\right)$ and transverse $\left(\mathrm{E}_{22}\right)$ directions, major Poisson's ratio $\left(v_{12}\right)$, and shear modulus $\left(\mathrm{G}_{12}\right)$. These four independent elastic constants are used to compute the non-zero matrix elements and describe the in-plane elastic behavior of a single orthotropic lamina in which $\theta=0$ or $90^{\circ}$ (Eq. 3.2). The stiffness matrix for a lamina with principal material axes at a $\theta$ other than 0 or $90^{\circ}$ is determined by applying a rotation transformation to the [Q] matrix [20].

$$
Q_{11}=\frac{E_{11}}{1-v_{12} v_{21}} \quad Q_{12}=\frac{v_{12} E_{22}}{1-v_{12} v_{21}}=\frac{v_{21} E_{11}}{1-v_{12} v_{21}}=Q_{21} \quad Q_{22}=\frac{E_{22}}{1-v_{12} v_{21}} \quad Q_{66}=G_{12}
$$

In applying CLT to FDM specimen analysis, we consider that the AM process forms parts by stacking individual laminae, each with principal material axes 1-2-3 (Figure 3a), and with reduced stiffness [Q] as previously described. The origin of the xyz axis associated with the entire laminate is located at the geometric midplane of the completed specimen, with the $x$-axis corresponding to the loading direction, the $\mathrm{x}-\mathrm{y}$ axes defining the build plane, and the $\mathrm{z}$ axis representing the thickness direction (Figure $3 \mathrm{~b}$ ).

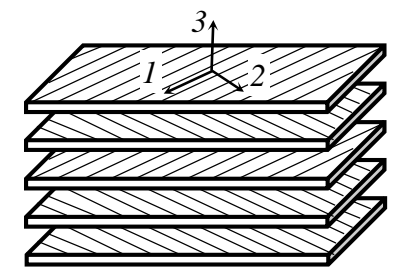

(a)

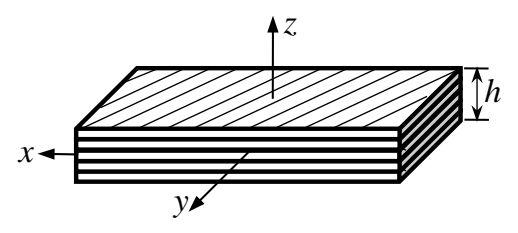

(b)

Fig. 3. FDM specimens as laminate composites; (a) The stacking of FDM layers or laminae to create a laminate, and (b) a completed FDM component or laminate.

Lamination theory [21] assumes a linear distribution of strain in the thickness direction, from which the resultant forces and moments are determined. With this, the force and moment equations for the full laminate are based on its extensional stiffness $(N / m)$ matrix [A], coupling stiffness $(N)$ matrix [B], and bending stiffness $(\mathrm{N} / \mathrm{m})$ matrix [D]. The elements of stiffness matrices [A], [B], and [D] depend on the elastic properties of each individual lamina, and its z-distance from the laminate midplane (x-y plane).

FDM specimens in this study had 15 layers each, and represent symmetric laminates i.e. for each lamina located above the midplane there is an identical lamina at an equal distance below the midplane. It can be shown, therefore, that there is no extension-bending coupling in the laminate, and $[\mathrm{B}]=[0]$. Therefore, the ability of the FDM specimens to resist in-plane loads is dictated by [A], while the capacity to resist bending and torsional loads is controlled by [D]. The elements of [A] are associated with lamina stiffness and thickness. For symmetric FDM laminates, [A] is a function of fiber material, fiber orientation angle, air void fraction, lamina thickness, and the number of layers of each orientation [18]. 
For a uniaxial tension load along $x$, the midplane elastic modulus of the laminate in the $x$-direction (axial) is given by Eq. 3.3:

$$
E_{x x}=\frac{1}{h\left[A^{-1}\right]_{11}}
$$

where $h$ is the full laminate thickness in the z-direction (Figure 3b) [18].

\subsection{Determination of Elastic Constants}

In order to calculate the extensional stiffness matrix [A] of a full laminate, the in-plane elastic behavior of each lamina is determined by its [Q] matrix. Each [Q] is computed from the elastic constants associated with the specially orthotropic case, i.e. $\mathrm{E}_{11}, \mathrm{E}_{22}, \mathrm{G}_{12}$, and $\mathrm{v}_{12}$ for $\theta=0$ and $90^{\circ}$, as estimated using experimental results. The longitudinal modulus $\mathrm{E}_{11}$ is determined using monotonic tension test results for the $0^{\circ}$ specimens, which have laminae composed of unidirectional fibers aligned with the loading axis $\left(0^{\circ}\right.$ in Figure 1b). Although full laminates of 15 layers, $E_{11}$ is estimated as the mean slope of the linear portions of the resulting stress-strain curves (Table 1). Major Poisson's ratio $\left(\mathrm{v}_{12}\right)$ is also determined with this loading configuration, as the negative ratio of the strains measured perpendicular and parallel to the loading axis. Similarly, $\mathrm{E}_{22}$ is computed as the mean slope of the linear portions of the stress-strain curves resulting from uniaxial tension tests of $90^{\circ}$ specimens. These specimens $\left(90^{\circ}\right.$ in Figure $\left.1 \mathrm{~b}\right)$ represent laminates in which each lamina is composed of fibers oriented perpendicular to the loading axis. Minor Poisson's ratio $\left(\mathrm{v}_{21}\right)$ is determined as the negative ratio of the strains measured perpendicular and parallel to the loading axis. Finally, $45^{\circ}$ specimens tested in uniaxial tension ( $45^{\circ}$ in Figure $1 \mathrm{~b}$ ) are used in computing the shear modulus, $\mathrm{G}_{12}$. The mean slope of the linear portions of the associated stress-strain curves provides an estimate of $\mathrm{E}_{x}\left(\right.$ or $\left.\mathrm{E}_{45^{\circ}}\right)$. With these elastic constants, the shear modulus is computed using transformation relations [20].

In this study, the experimentally based estimates of $\mathrm{E}_{11}, \mathrm{E}_{22}, \mathrm{G}_{12}$, and $\mathrm{v}_{12}$ and $\mathrm{Eq} .3 .2$ produce a reduced stiffness matrix [Q] with $\mathrm{Q}_{11}=1643.8, \mathrm{Q}_{12}=426.3=\mathrm{Q}_{21}, \mathrm{Q}_{22}=1152.2, \mathrm{Q}_{66}=370.4\left(\mathrm{~N} / \mathrm{m}^{2}\right)$. [Q] is then used to compute [A] and the effective elastic modulus $E_{x x}$ for a symmetric specimen associated with each of the mesostructures tested in monotonic tension. Figure 4 displays experimental values for mean effective modulus and those obtained using the described CLT method.

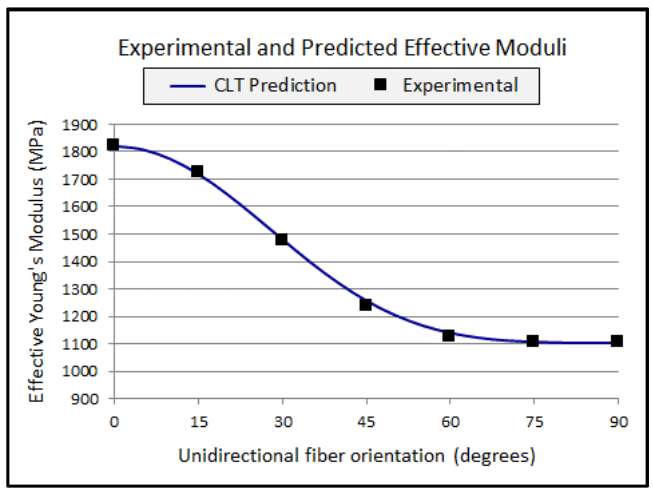

(a)

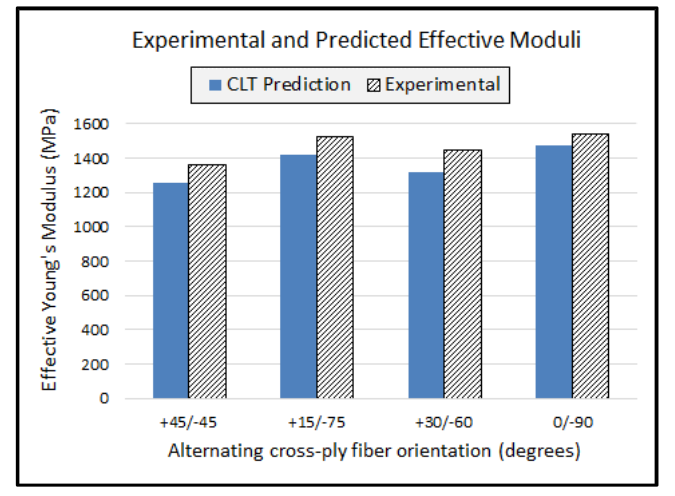

(b)

Fig. 4. Comparisons of experimental and CLT predicted mean effective moduli, for FDM laminates with mesostructures of (a) unidirectional parallel plies and (b) bidirectional orthogonal plies.

\section{Fatigue Damage Analysis}

The agreement between the experimental effective moduli of the specimens and the predicted values obtained using CLT (Figure 4) suggests behavioral similarities with regard to the elastic tensile performance of FDM parts and that of more traditional composite laminate structures. If such similarities 
extend to cyclical performance, then complex phenomena associated with FDM fatigue damage can be expected to affect specimen strength and stiffness [22]. The literature is rich in studies demonstrating elastic modulus degradation and its use in quantifying the cyclical damage of composite laminate materials [23-28]. Such work includes efforts to formulate models to predict the damage mode and the delamination initiation and propagation under fatigue cycling. For a composite structure, however, the multiple damage modes also interact with each other, further complicating attempts at a mechanistic solution. A similar understanding of the cyclical damage evolution of AM parts is important to serve as the foundation for future modeling and prediction of fatigue life for engineering applications.

\subsection{Modulus degradation during cyclical loading}

In this study, the stiffness degradation of cyclically loaded FDM specimens was evaluated as a function of component mesostructure and maximum stress level. The effective elastic modulus for each specimen was calculated from the slope of the linear portion of the stress-strain curves. A least squares linear regression analysis of the data was used to compute the effective elastic modulus $E(n)$ for each cycle $n$ in the fatigue life $N$ of a specimen. The change in magnitude of $E(n)$, as cycle $n$ increased, was analyzed for: (a) a given mesostructure, with different maximum stress levels, and (b) a given maximum stress level, for various mesostructures. The effective modulus and the number of cycles were normalized to allow for more meaningful comparisons to be made across mesostructures and stress levels. Normalized effective modulus $E_{n}(n)=E(n) / E(1)=E(n) / E_{o}$, (where $E_{o}$ is the original modulus) and normalized cycle number $=n / N$.

Figure 5 displays the trends in stiffness degradation for each bidirectional mesostructure, with different maximum stress levels.

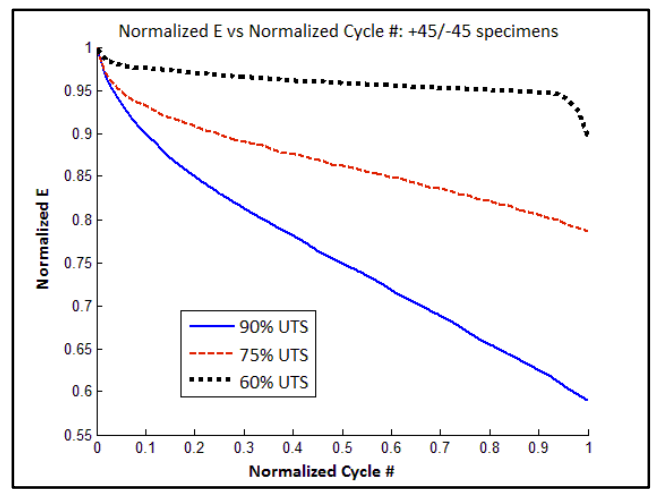

(a)

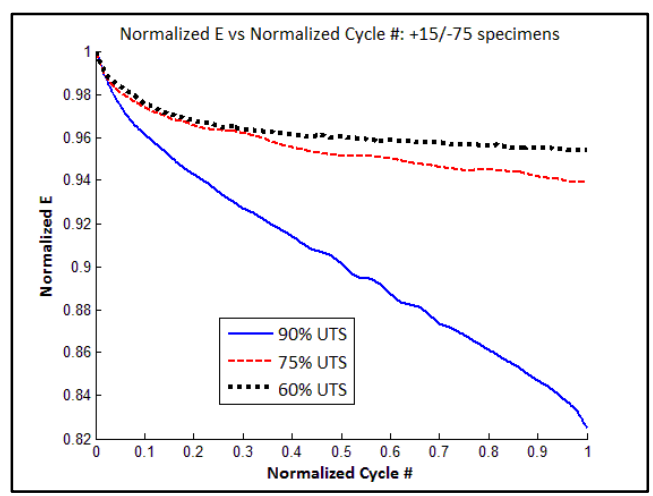

(c)

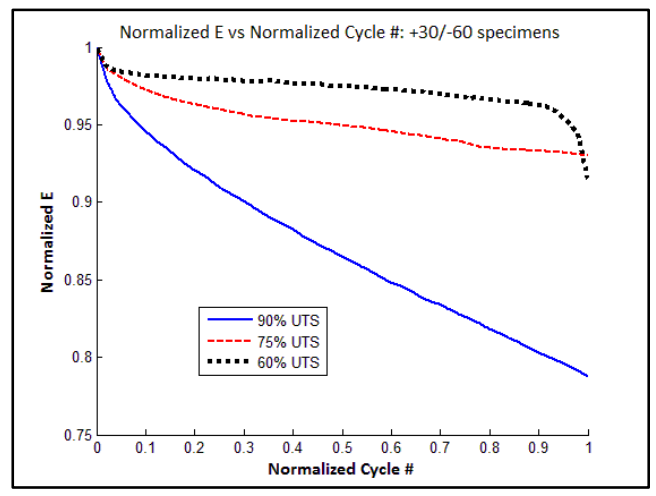

(b)

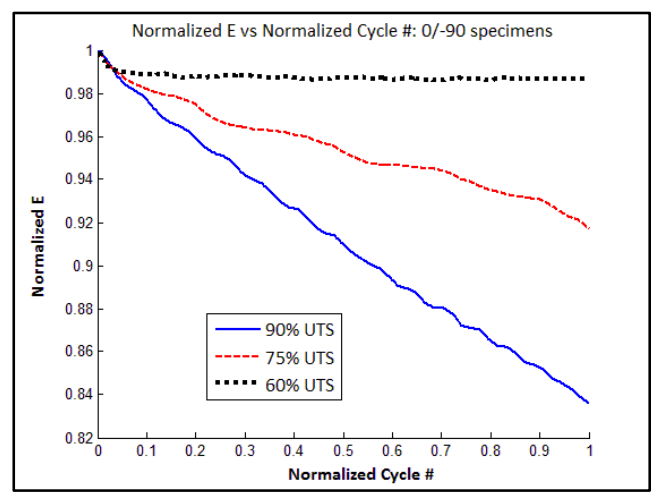

(d)

Fig. 5. Plots of $n / N$-vs- $E(n) / E_{o}$ for representative bidirectional specimens at different maximum stress levels. Mesostructures of: (a) $+45^{\circ} /-45^{\circ}$, (b) $+30^{\circ} \%-60^{\circ}$, (c) $+15^{\circ} /-75^{\circ}$, (d) $0 \%-90^{\circ}$. 
All four mesostructures display the highest rate of stiffness degradation $d E / d n$ when cycled with the highest maximum stress (90\% UTS). In addition, at each stress level, each mesostructure experiences its highest rate of stiffness degradation in the first 5-10\% of its fatigue life. Several of the mesostructure/stress level combinations also experience another steep degradation rate in the last 5-10\% of its fatigue life. The overall degradation of normalized effective modulus is most significant for the $+45 /-45 @ 90 \%$ UTS, for which the mean minimum value of $E_{n}(N)=0.59$.

Figure 6 displays the trends in stiffness degradation for each of the unidirectional mesostructures studied. The degradation rates of these specimens do not present the same obvious dependence on loading level that is seen in the bidirectional components. This is most apparent in considering the $45^{\circ}$ and $90^{\circ}$ mesostructures (Figures $6 \mathrm{~b}$ and $6 \mathrm{c}$ ). This is in part due to the fact that the range of normalized $E$ values is significantly smaller than that seen in the bidirectional specimens; indicating that the percentage change in stiffness is not as great over the fatigue life of these specimens. For instance, the minimum value of $E_{n}(N)$ was closer to 0.77 , or a $23 \%$ stiffness loss. In addition, the difference between $E_{n}(N)$ across the three \%UTS values is significantly smaller in the unidirectional (maximum of 0.12 for $0^{\circ}$ ) than the bidirectional (maximum of 0.34 for $+45^{\circ} /-45^{\circ}$ ) specimens. Also of relevance is the fact that the $45^{\circ}$ and $90^{\circ}$ specimens have much shorter mean fatigue lives than the other specimens, as seen in Table 2 . It is possible that the shorter fatigue lives, the smaller overall change in specimen stiffness, and the absence of any obvious influence of \%UTS on stiffness degradation are all affected by the dependence of the $45^{\circ}$ and $90^{\circ}$ mesostructures on inter-fiber bonding, which is a consequence of the manufacturing process. As highlighted in a previous study [12], the interfiber bond is significantly weaker than the ABS monofilament itself, and has more inherent variability as a result of the FDM extrusion process.

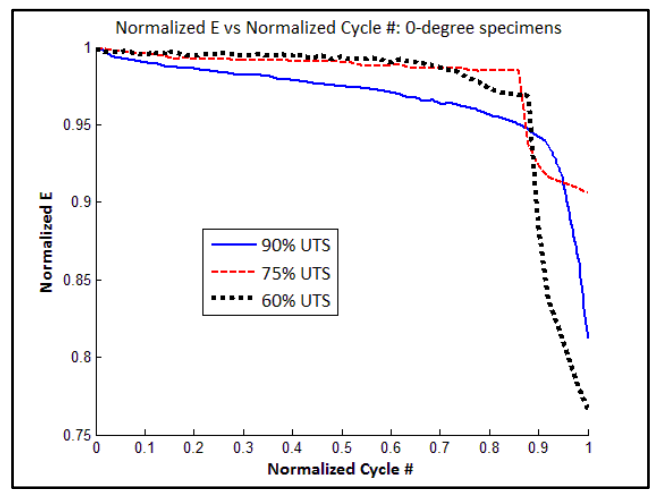

(a)

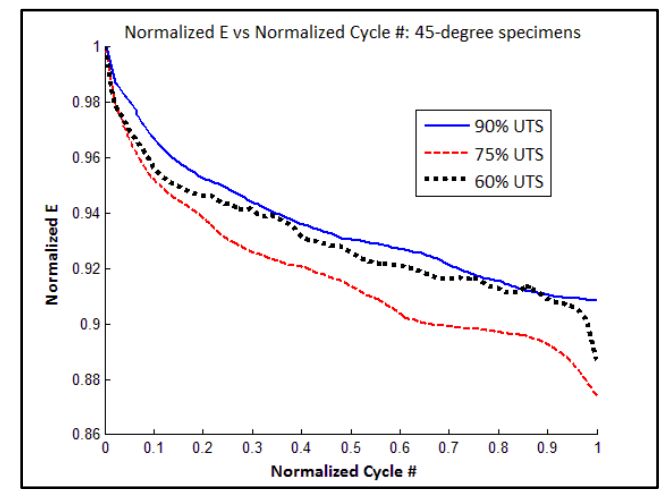

(b)

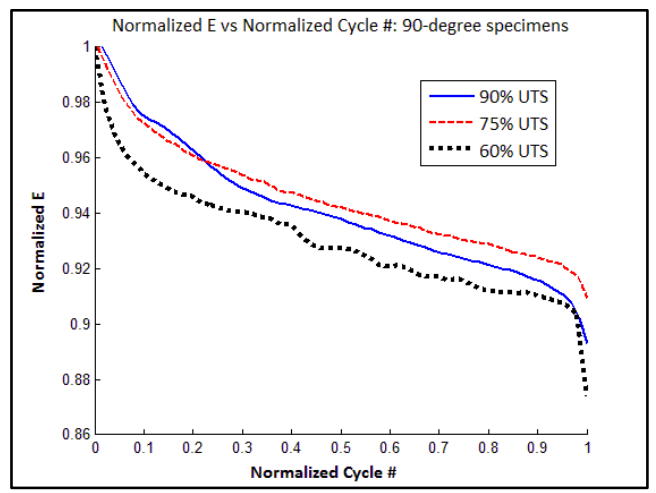

(c)

Fig. 6. Plots of $n / N$-vs- $E(n) / E_{o}$ for representative unidirectional specimens at different maximum stress levels, with mesostructures of: (a) $0^{\circ}$, (b) $45^{\circ}$, (c) $90^{\circ}$. 


\subsection{Fatigue Damage Accumulation}

Knowledge of the degradation of stiffness is often used to estimate the damage state, $D$, of traditional laminate composites for cases in which constant cyclical load amplitude is applied and consistent stiffness measurements are performed [28]. Damage is known to accumulate nonlinearly in composite materials, and is typically described as having three stages, i.e. rapid damage accumulation during the first few cycles, slow and steady damage rate during the longest middle phase of fatigue life, and another rapid rate of damage growth in the final few cycles. Given that the experimental results obtained in this work demonstrate three similar phases with regard to the rate of stiffness degradation (Figure 6), and the measured effective modulus just prior to failure is not zero (i.e. $E_{f} \neq 0$ ), an expression for the damage state based on $E_{f}$ was expected to be valid. Specifically, analysis of the normalized damage state $D$ of an FDM specimen at any cycle $n$ of cyclical loading was analyzed here as the quotient of the stiffness degradation at cycle $n$ divided by the total stiffness degradation at time of failure (Eq. 4.1):

$$
D(n)=\frac{E_{o}-E(n)}{E_{o}-E_{f}}
$$

where $E_{o}$ is the original modulus (at cycle 1), and $E_{f}$ is the modulus when fracture occurs. This damage state was first introduced to quantify the accumulated damage in fiber-reinforced composite materials [23]. As defined in Eq. 4.1, the range of $D(n)$ is [0,1] for all fatigue tests that continue until failure.

The initially high rate of stiffness degradation seen in the first and third phases of fatigue cycling (Figures 6), can now be observed in terms of the progressive damage state. Because the range of damage state $D(n)$ is $[0,1]$ for all tests, the $n / N-v s-D(n)$ plots are further normalized, permitting generalized comparisons across multiple mesostructures and loading cases. Figure 7 displays the comparison of $n / N$ $v s-E(n) / E_{o}$ and $n / N-v s-D(n)$ plots for the same specimens as an example. The general shape of Figure 7(b) resembles the cyclical behavior of composite materials with multiple plies and laminates [28]. Similar to composites, FDM specimens appear to experience multiple damage modes relatively early in their fatigue life. Damage modes observed during the SEM inspection of the failure surfaces of cyclically loaded FDM specimens include crazing, fiber cracking, delamination, and void changes (Figure 8).

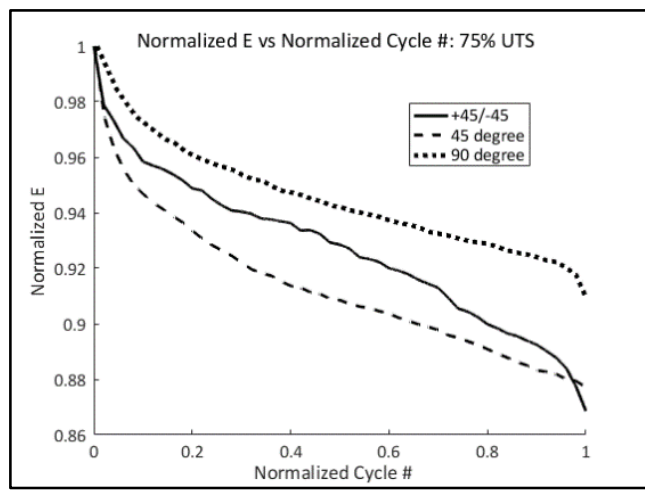

(a)

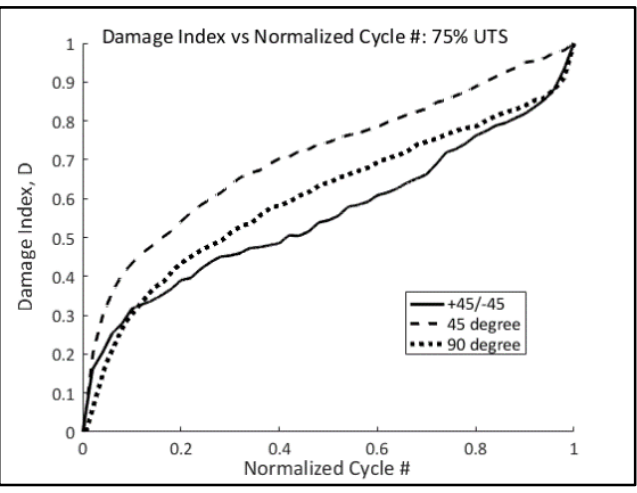

(b)

Fig. 7. Cyclical behaviour of representative specimens with $+45 /-45^{\circ}, 45^{\circ}$, and $90^{\circ}$ mesostructures at $75 \%$ UTS maximum loading level; (a) $n / N-v s-E(n) / E_{o}$; (b) $n / N-v s-D(n)$;

\subsection{Fatigue Damage Model}

The form of an analytical model for damage accumulation used in this work is one proposed by Mao and Mahadevan [23], based on continuum damage mechanics, as follows:

$$
D_{a}(n)=q\left(\frac{n}{N}\right)^{m_{1}}+(1-q)\left(\frac{n}{N}\right)^{m_{2}}
$$



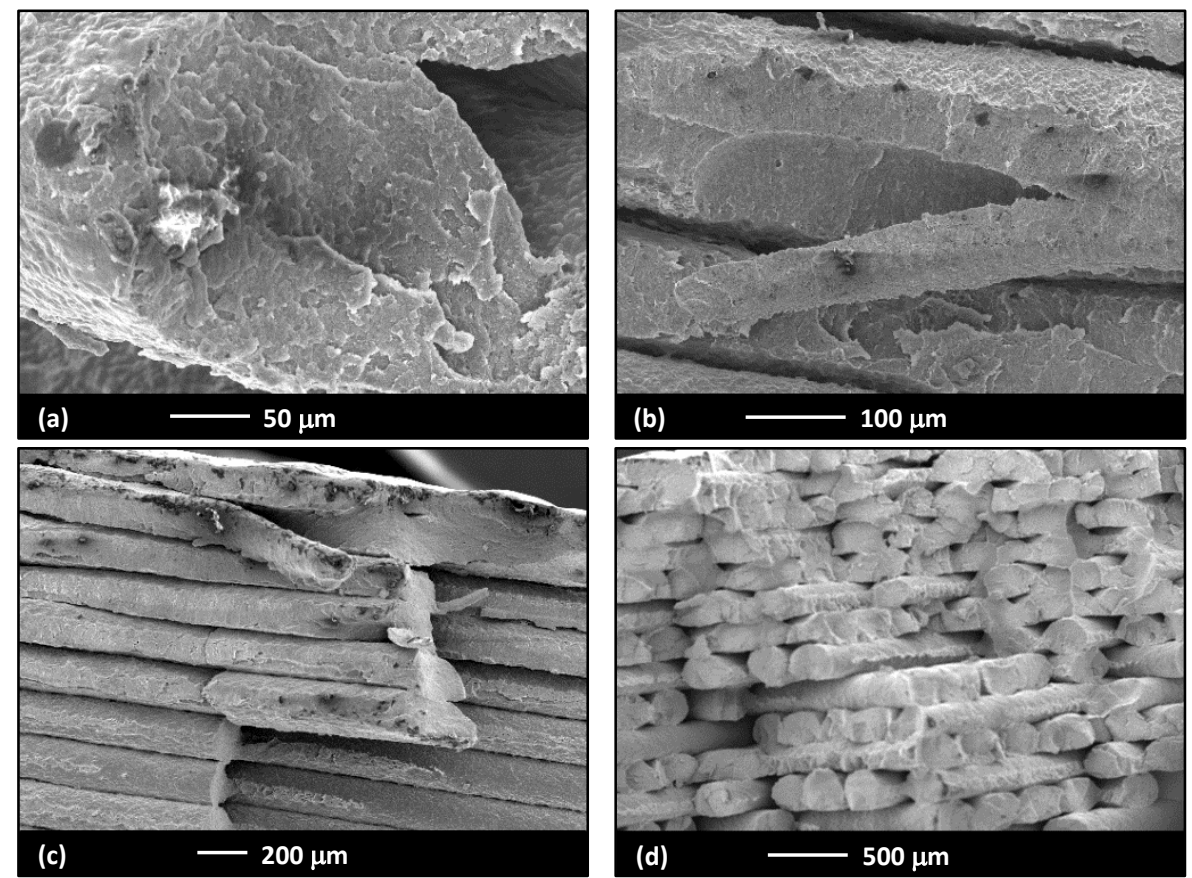

Fig. 8. SEM micrographs of fatigue fracture surfaces, displaying damage modes of: (a) crazing, (b) fiber cracking, (c) delamination, and (d) void changes.

$D_{a}(n)$ is the analytical expression for normalized accumulated damage at cycle $n ; q, m_{1}$, and $m_{2}$ are parameters that depend on FDM specimen mesostructure and load level; $n$ is the number of applied loading cycles; and $N$ is the fatigue life of the specimen at the corresponding applied loading level. The first term in this expression is useful in capturing the characteristics of the rapid damage accumulation that seems to occur in the early cycles of loading, with $m_{l}<1$. The second term models the quick damage growth that often occurs at the end of fatigue life, with $m_{2}>1$. The $q$ parameter serves as a weighting factor for these different trends.

In this study, the values for $q, m_{1}$, and $m_{2}$ are extracted by means of a least squares nonlinear regression analysis of damage states for each factor combination of mesostructure and load level tested. In each case, all data points were used in Eq. 4.1 to obtain a cloud of damage states, $D(n)$, and the experimental curves were fitted to obtain the model parameters. The results are displayed in Table 4, together with the associated coefficients of determination $\left(\mathrm{R}^{2}\right)$ as a measure of fit quality.

The results from the cyclical loading of unidirectional $\left(90^{\circ}\right)$ and bidirectional $\left(+45 /-45^{\circ}\right)$ mesostructures are used here to graphically demonstrate the use of the proposed damage accumulation function to model the stiffness degradation of FDM parts. The behavior of the two mesostructures is considered at three different maximum loading levels, and the experimental results are plotted together with the proposed model in Figure 9. It can be seen that the proposed function models the experimental results very well, with the mean coefficient of determination, $R^{2}$, equal to 0.998 for $+45 /-45^{\circ}$ and 0.987 for $90^{\circ}$.

It can be noted by examination of the results in Table 4 that the values for $q$ and $m_{l}$ are highest for the $90 \%$ UTS loading case and lowest for the $60 \%$ UTS case for all mesostructures tested other than the unidirectional $0^{\circ}$ specimens. This trend in parameter values is associated with the progression of damage curve shapes seen in Figure 9, from left to right. The 60\% UTS cases appear to display the threephased shape of traditional composites, i.e. a steep rates of damage accumulation in the first and last $5 \%$ of fatigue life, and a gradual rate over the center $90 \%$ of life [28]. These plot appear in Figure 9c and 9f. 
Table 4. Estimated parameters resulting from curve fitting for the proposed damage accumulation model

\begin{tabular}{cccccc}
\hline $\begin{array}{c}\text { Fiber } \theta \\
\text { Mesostructure }\end{array}$ & $\begin{array}{c}\sigma_{\max } \\
(\% \mathrm{UTS})\end{array}$ & $q$ & $m_{1}$ & $m_{2}$ & $\mathrm{R}^{2}$ \\
\hline $0^{\circ}$ & 90 & 0.252 & 0.810 & 22.568 & 0.9958 \\
& 75 & 0.161 & 0.268 & 196.881 & 0.7718 \\
& 60 & 0.821 & 0.844 & 12.178 & 0.9137 \\
\hline $45^{\circ}$ & 90 & 1.000 & 0.429 & - & 0.9902 \\
& 75 & 0.900 & 0.375 & 31.685 & 0.9909 \\
& 60 & 0.837 & 0.361 & 55.537 & 0.9919 \\
\hline $90^{\circ}$ & 90 & 0.815 & 0.469 & 31.855 & 0.9790 \\
& 75 & 0.875 & 0.449 & 42.022 & 0.9920 \\
& 60 & 0.740 & 0.338 & 74.933 & 0.9885 \\
\hline $0^{\circ} /-90^{\circ}$ & 90 & 0.991 & 0.850 & 62.704 & 0.9995 \\
& 75 & 0.867 & 0.610 & 10.707 & 0.9954 \\
& 60 & 0.480 & 0.489 & 3.442 & 0.9752 \\
\hline$+15^{\circ} / 75^{\circ}$ & 90 & 0.867 & 0.608 & 6.588 & 0.9991 \\
& 75 & 0.928 & 0.393 & 62.839 & 0.9891 \\
& 60 & 0.636 & 0.280 & 18.208 & 0.9839 \\
\hline$+30^{\circ} / 60^{\circ}$ & 90 & 0.897 & 0.624 & 5.178 & 0.9994 \\
& 75 & 0.962 & 0.388 & 3.515 & 0.9940 \\
& 60 & 0.404 & 0.331 & 29.267 & 0.9670 \\
\hline$+45^{\circ} /-45^{\circ}$ & 90 & 0.877 & 0.551 & 3.127 & 0.9996 \\
& 75 & 0.853 & 0.424 & 3.023 & 0.9991 \\
& 60 & 0.528 & 0.362 & 45.620 & 0.9960 \\
\hline
\end{tabular}

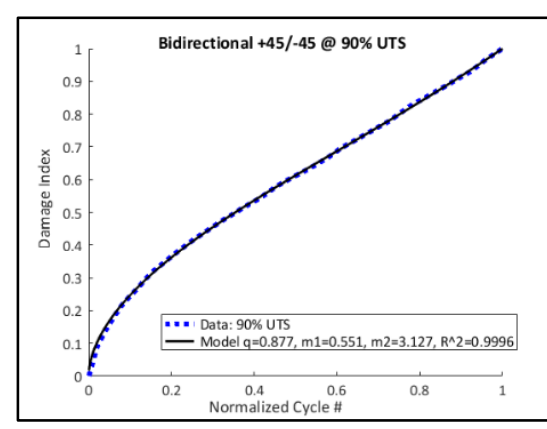

(a)

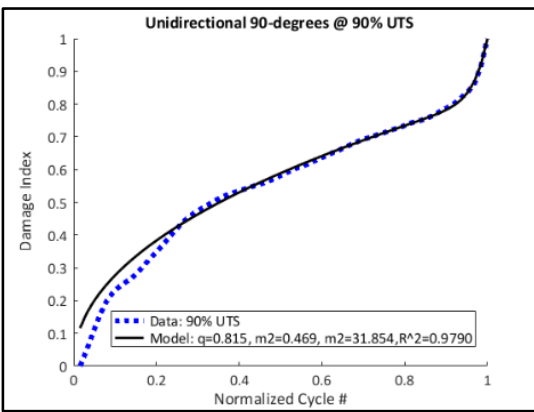

(d)

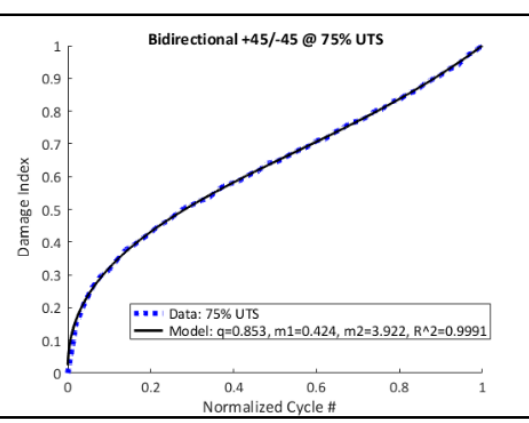

(b)

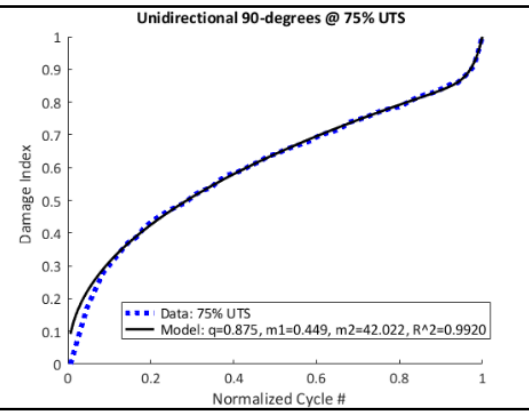

(e)

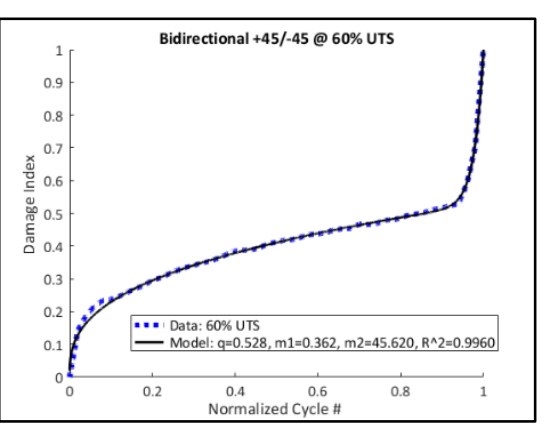

(c)

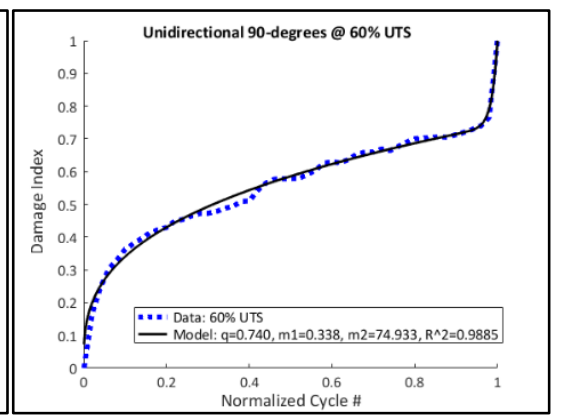

(f)

Fig. 9. Damage evolution model curves and experimental data for $+45 \%-45^{\circ}$ mesostructure at (a) $90 \%$, (b) $75 \%$, (c) $60 \%$ UTS, and $90^{\circ}$ mesostructure at (d) $90 \%$, (e) $75 \%$, (f) $60 \%$ UTS. 
Another interesting result appears with cases in which a high rate of damage accumulation in the first 2$3 \%$ of fatigue life is not followed by a subsequent high rate of damage at the end of fatigue life. This behavior was seen in several individual FDM specimens, and results in $q$ being very close to 1.0. Serving as a weighting factor in the model (Eq. 4.2), it can be seen that a $q$-value of approximately 1.0 causes the second term of the function and the value of $m_{2}$ to become insignificant; inherently changing the shape of the function. An example of this damage accumulation situation is seen in Table 4 for the $90 \%$ UTS case of the $45^{\circ}$ mesostructure. Displayed graphically in Figure 10, it can be seen that there is even a slight decrease in the rate of damage accumulation detectable in the final 2-3\% of the fatigue life in this case, although the model as defined is unable to capture this decreasing slope trend.

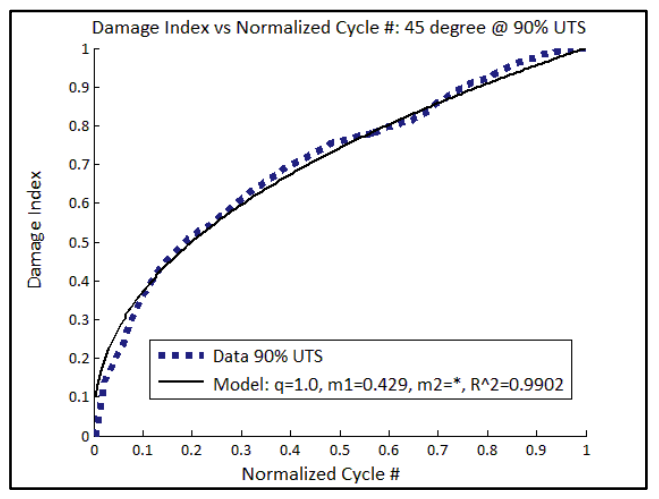

Fig. 10. Damage evolution model curve and experimental data for $+45^{\circ}$ mesostructure at $90 \%$ UTS.

\section{Conclusions}

This work is believed to be the first documented study to investigate the characteristics of fatigue damage accumulation in FDM specimens. Cyclical tension fatigue tests were conducted and the resulting degradation of the effective Young's modulus, $E$, was analyzed for multiple mesostructure/loading combinations, to determine if the fatigue behavior emulated that of more traditional composite laminates. The following conclusions have been drawn:

(a) The cross-ply bidirectional specimens experience three different stages of damage evolution of under cyclical loading; similar to the damage trends seen in fiber reinforced composites. Fatigue damage grows rapidly in the first stage (i.e. first 2-3\% of fatigue life) due to the occurrence of multiple damage modes, such as crazing, fiber cracking, delamination, void geometry changes. The damage then increases steadily and slowly during the second and longest stage of fatigue life. In final stage includes the rapid damage due to fiber fracture.

(b) The unidirectional $0^{\circ}$ parts did not display this same pattern of damage. This suggests that the $0^{\circ}$ parts are most influenced by the behavior of the aligned polymer fibers themselves, which is in agreement with their monotonic tensile behavior [12].

(c) A nonlinear damage accumulation model, originally proposed for fiber reinforced composites, effectively captures the unique characteristics of the damage evolution seen in FDM specimens with bidirectional mesostructures subjected to cyclical loading.

The ability to accurately model the accumulated damage of FDM specimens is crucial for fatigue life prediction. While the proposed model appears to accomplish this well, with $\mathrm{R}^{2}$ values between 0.9670 and $0.9996\left(0^{\circ}\right.$ parts excluded $)$, future work will need to carefully consider the less predictable damage trend 
that occurs in the last $5 \%$ of fatigue life. It is also important to recognize that the design of FDM components would logically avoid consideration of the final $5 \%$ of fatigue life due to the desire to include a design factor of safety. In this case, the damage model may be more useful if based on the experimental data from $95 \%$ of total fatigue life; essentially removing consideration of the steep damage rate that often occurs in the final few cycles.

Finally, the fatigue damage mechanisms described above are also believed to cause changes in the residual strength of FDM specimens. Therefore, work is ongoing to consider the accumulation of damage on the basis of the degradation of residual strength. Damage states based on strength will be determined, and an associated damage model will be compared with the results of this work.

\section{Acknowledgements}

The authors acknowledge the funding provided by the National Science Foundation, DUE-1317446, and the STEM Scholars program.

\section{References}

[1] Negi, S., Dhiman, S., Sharma, R. (2013). Basics, Applications and Future of Additive Manufacturing Technologies: A Review, Journal of Manufacturing Technology Research, Vol. 5, No. 1/2, pp. 7596.

[2] Fernández, S., Jiménez, M., Porras, J., Romero, L., Espinosa, M., Domínguez, M. (2016). Additive Manufacturing and Performance of Functional Hydraulic Pump Impellers in Fused Deposition Modeling Technology, Journal of Mechanical Design, Vol. 138, No. 2, pp. 024501-1 - 024501-4.

[3] Rodriguez, J., Thomas, J. and Renaud, J. (2003). Mechanical behavior of acrylonitrile butadiene styrene fused deposition materials modeling, Rapid Prototyping Journal, Vol. 9, No. 4, pp. 219-230.

[4] Ahn, S., Montero, M., Odell, D., Roundy, S. and Wright, P. (2002). Anisotropic Material Properties of Fused Deposition Modeling ABS, Rapid Prototyping Journal, Vol. 8, No. 4, pp. 248 -257.

[5] Sood A., Ohdar R. and Mahapatra, S. (2010). Parametric appraisal of mechanical property of fused deposition modelling processed parts, Materials and Design, Vol. 31, No. 1, pp. 287-95.

[6] Es Said, O., Foyos, J., Noorani, R., Mendelson, M., Marloth, R. and Pregger, B. (2000). Effect of layer orientation on mechanical properties of rapid prototyped samples, Materials and Manufacturing Processes, Vol. 15, No. 1, pp. 107-22.

[7] Ziemian, C., Sharma, M., Ziemian, S. (2012), "Anisotropic Mechanical Properties of ABS Parts Fabricated by Fused Deposition Modelling”, In: Mechanical Engineering, Dr. Murat Gokcek (Ed.), ISBN: 978-953-51-0505-3, InTech.

[8] Sood, A., Ohdar, R. \& Mahapatra, S. (2012). Experimental investigation and empirical modeling of FDM process for compressive strength improvement, Journal of Advanced Research, Vol. 3, No. 1, pp. 81-90.

[9] Lee, C., Kim, S., Kim, H. and Ahn, S. (2007). "Measurement of anisotropic compressive strength of rapid prototyping parts", Journal of Materials Processing Technology, Vol. 187-188, pp. 627-630.

[10] Ziemian, S.N., Okwara, M.N., Ziemian, C.W. (2015). Tensile and Fatigue Behavior of Layered Acrylonitrile Butadiene Styrene, Rapid Prototyping Journal, Vol. 21, No. 3, pp 270 - 278.

[11] Lee, J. and Huang, A. (2013), Fatigue analysis of FDM materials, Rapid Prototyping Journal, Vol. 19, No. 4, pp. 291-299.

[12] Ziemian, C. W., Cipoletti, D. E., Ziemian, S. N., Okwara, M.N., Haile, K.V. (2014). Monotonic and Cyclic Tensile Properties of ABS Components Fabricated by Additive Manufacturing, Proceedings of 25th International Solid Freeform Fabrication Symposium, Austin, Texas, August 4-6. 
[13] Caprino, G. (2000). Predicting Fatigue Life of Composite Laminates Subjected to Tension-Tension Fatigue, Journal of Composite Materials, Vol. 34, No. 16, pp. 1334-1355.

[14] ASTM Standard D638 - 10. (2010). Standard Test Method for Tensile Properties of Plastics. ASTM International, West Conshohocken, Pennsylvania, DOI: 10.1520/D0638-10, Available from: www.astm.org

[15] ASTM Standard D7791 - 12. (2012). Standard Test Method for Uniaxial Fatigue Properties of Plastics. ASTM International, West Conshohocken, Pennsylvania, DOI: 10.1520/D7791-12, Available from: www.astm.org

[16] Antony, J. (2014). Design of Experiments for Engineers and Scientists, Second Edition. Elsevier Science and Technology Books, Inc.

[17] Kulkarni, P., Dutta, D. (1999). Deposition Strategies and Resulting Part Stiffnesses in Fused Deposition Modeling, Journal of Manufacturing Science and Engineering, Vol. 121, pp. 93-103.

[18] Li, L., Sun, Q., Bellehumeur, C., Gu, P. (2002). Composite Modeling and Analysis for Fabrication of FDM Prototypes with Locally Controlled Properties, Journal of Manufacturing Processes, Vol. 4(2), pp. 129-141.

[19] Casavola, C., Cazzato, A., Moramarco, V., Pappalettere, C. (2016). Orthotropic mechanical properties of fused deposition modelling parts described by classical laminate theory, Materials and Design, Vol. 90, pp. 453-458.

[20] Robert M. Jones. (1998). Mechanics of Composite Materials, Materials Science and Engineering Series, CRC Press, ISBN 9781560327127.

[21] P.K. Mallick, (2007). Fiber-Reinforced Composites: Materials, Manufacturing, and Design, Third Edition, CRC Press, ISBN 9780849342059

[22] Shiri, S., Yazdani, M., Pourgol-Mohammad, M. (2015). A fatigue damage accumulation model based on stiffness degradation of composite materials, Materials \& Design, Vol. 88, pp. 1290 1295 .

[23] Mao, H., Mahadevan, S. (2002). Fatigue damage modelling of composite materials, Composite Structures, Vol. 58, pp. 405-410.

[24] Yang, J.N., Lee, L.J., Sheu, D.Y. (1992). Modulus reduction and fatigue damage of matrix dominated composite laminates, Composite Structures, Vol. 21, pp. 91-100.

[25] Mortell, D., Tanner, D., McCarthy, C. (2016). An experimental investigation into multi-scale damage progression in laminated composites in bending, Composite Structures, Vol. 149, pp. 33-40.

[26] Yang, J.N., Jones, D.L., Yang, S.H., Meskini, A. (1990). A Stiffness Degradation Model for Graphite/Epoxy Laminates, Journal of Composite Materials, Vol. 24, pp. 753-769. DOI: $10.1177 / 002199839002400705$.

[27] Zhang, W., Zhou, Z., Scarpa, F., Zhao, S. (2016). A fatigue damage meso-model for fiber-reinforced composites with stress ratio effect, Materials \& Design, Vol. 107, pp. 212 - 220

[28] Giancane, S, Panella, F, Dattoma, V. (2010). Characterization of fatigue damage in long fiber epoxy composite laminates, International Journal of Fatigue, Vol. 32, pp. 46-53. 
FDM specimens;

Multiple mesostructures

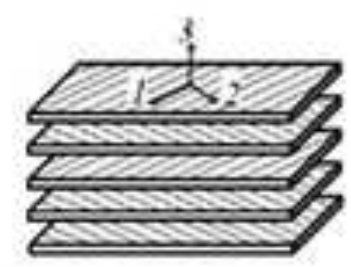

Fiber orientations \& layering pattern

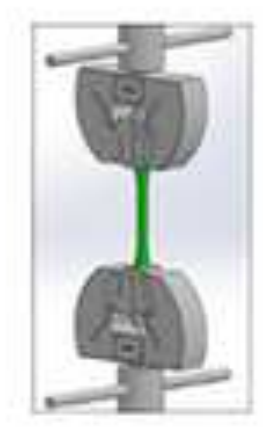

Cyclical loading to failure

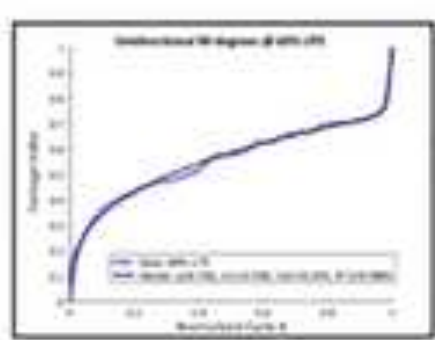

Model of stiffness-

based damage state

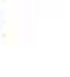

\section{of stiffness, $E$}

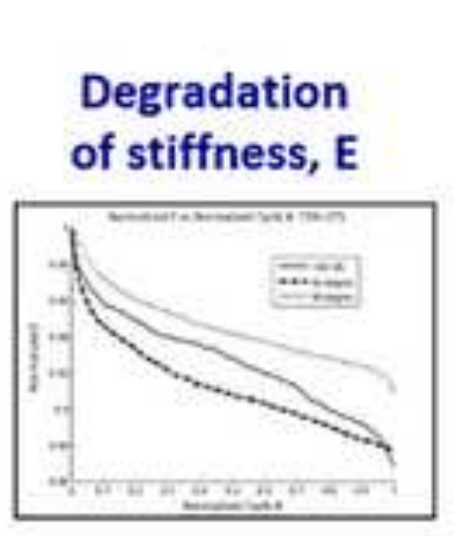

(1)

